\title{
No evidence of a synergistic effect of HIV infection and diabetes mellitus type 2 on fat distribution, plasma adiponectin or inflammatory markers
}

Malene Hove-Skovsgaard', Julie Abildgaard², Marco Gelpi ${ }^{1}$, Julie Christine Gaardbo ${ }^{1}$, Lilian Kolte ${ }^{3,4}$, Henrik Ullum ${ }^{5}$, Marius Trøseid ${ }^{6,7,8}$, Birgitte Lindegaard ${ }^{2,4}$ and Susanne Dam Nielsen ${ }^{1 *}$ (D)

\begin{abstract}
Background: Altered fat distribution and chronic inflammation are found in both persons living with HIV (PLWH) and persons with diabetes mellitus type 2 (DM2) and are known risk factors for cardiovascular diseases (CVD). We aimed to investigate if a synergistic effect of HIV infection and DM2 was found on fat distribution and inflammation.

Methods: A cross-sectional study was performed including PLWH with HIV RNA < 200 copies/mL (18 with DM2 (HIV + DM2+), 18 without DM2 (HIV + DM2-)) and controls (19 with DM2 (controls with DM2) and 25 without DM2 (healthy controls). We measured fat distribution using dual-energy $X$-ray absorptiometry scan. Plasma concentrations of adiponectin, interleukin-6 (IL-6), tumor necrosis factor-alfa (TNF- $a$ ) and soluble CD14 (sCD14) was measured using snap-frozen plasma.

Results: HIV + DM2 + and HIV + DM2- had comparable trunk/limb fat ratio. In contrast, HIV + DM2+ had a higher trunk/ limb fat ratio than controls with DM2 and healthy controls ( $p=0.013$ and $p<0.001$, respectively). However, HIV + DM2+ and controls with DM2 had comparable amount of trunk fat mass $(\mathrm{kg})(p=0.254)$. A lower concentration of plasma adiponectin and higher concentration of IL-6 was found in HIV + DM2+ than in HIV + DM2-( $p=0.037$ and $p=0.039)$ and in healthy controls ( $p=0.001$ and $p=0.012$ ). In contrast, plasma adiponectin and IL-6 concentrations were comparable in HIV + DM2+ and controls with DM2 ( $p=0.345$ and $p=0.825)$. Concentration of sCD14 was comparable in HIV + DM2+ and HIV + DM2- $(p=0.850)$, but elevated in HIV + DM2+ compared to controls with DM2 $(p<0.001)$ and healthy controls $(p=0.007)$. No statistical interactions were found between HIV infection and DM2 for any of the depending variables.

Conclusion: A synergistic effect of HIV and DM2 was not found for any of the outcomes. However, HIV + DM2+ had features related to both HIV infection and DM2 with a high trunk/limb ratio, high trunk fat mass, low concentration of plasma adiponectin and elevated concentrations of IL-6 and sCD14. This could contribute to elevated risk of CVD.
\end{abstract}

Keywords: HIV infection, Diabetes mellitus type 2, Fat distribution, Adiponectin, IL-6, SCD14

* Correspondence: sdn@dadlnet.dk

'Department of Infectious Diseases, Rigshospitalet, Copenhagen University Hospital, Copenhagen, Denmark

Full list of author information is available at the end of the article

(c) The Author(s). 2020 Open Access This article is licensed under a Creative Commons Attribution 4.0 International License, which permits use, sharing, adaptation, distribution and reproduction in any medium or format, as long as you give appropriate credit to the original author(s) and the source, provide a link to the Creative Commons licence, and indicate if changes were made. The images or other third party material in this article are included in the article's Creative Commons licence, unless indicated otherwise in a credit line to the material. If material is not included in the article's Creative Commons licence and your intended use is not permitted by statutory regulation or exceeds the permitted use, you will need to obtain permission directly from the copyright holder. To view a copy of this licence, visit http://creativecommons.org/licenses/by/4.0/ The Creative Commons Public Domain Dedication waiver (http://creativecommons.org/publicdomain/zero/1.0/) applies to the data made available in this article, unless otherwise stated in a credit line to the data. 


\section{Background}

After the introduction of combination antiretroviral treatment (cART), morbidity and mortality related to acquired immune deficiency syndrome (AIDS) have decreased [1]. However, a gap in life expectancy remains [2], and new challenges related to non-AIDS comorbidity have emerged including excess risk of cardiovascular disease (CVD) [3, 4].

Altered body composition with redistribution of body fat from subcutaneous departments to the visceral department is recognized as a common complication in persons living with HIV (PLWH) and especially in those exposed to older generations of cART including thymidine analogues [5]. Recently, weight gain and obesity in PLWH on modern cART regimes have been recognized as an increasing problem [6]. As in the general population weight gain and obesity is associated with both CVD and DM2 in PLWH $[7,8]$.

Adiponectin is an adipokine with anti-inflammatory properties. Both lipoatrophy and central fat accumulation have been associated with lower concentrations of plasma adiponectin in PLWH [9], and adiponectin could be a link between altered fat accumulation and DM2. Thus, low plasma adiponectin concentration has been associated with increased risk of DM2 in the general population [10], and CVD in both PLWH and in uninfected persons with DM2 [11, 12].

Human immunodeficiency virus infection is characterized by chronic inflammation, and even in persons with undetectable viral replication evidence of inflammation is found, when compared to uninfected controls [13, 14]. Likewise, DM2 is associated with increased inflammation [15].

The aim of this study was to investigate the combined effect of HIV and DM2 on fat distribution and inflammation. We hypothesized that PLWH with DM2 (HIV + $\mathrm{DM} 2+$ ) would have altered fat distribution with more trunk fat mass and less limb fat mass than PLWH without DM2 (HIV + DM2-), controls with DM2, and healthy controls. Also, we hypothesized that plasma adiponectin concentration would be lower and concentrations of IL-6, TNF- $\alpha$, and sCD14 would be higher in $\mathrm{HIV}+\mathrm{DM} 2+$ than in the three control groups indicating a synergistic effect of HIV infection and DM2.

\section{Methods}

\section{Participants}

This cross-sectional study was a part of a larger study entitled "HIV-infected persons with type 2 diabetes show evidence of endothelial dysfunction and increased inflammation" concerning inflammation and endothelial function in PLWH with DM2 [16]. Participants were included between August 2012 and August 2013. In total 100 participants were included in the primary study. When included all participants were invited to a Dual- energy X-ray absorptiometry scan (DXA-scan) which were optional since the participants had to make an additional visit to the hospital for this scan. In total 80 participants agreed to this (18 with HIV + DM2+, 18 with HIV + DM2-, 19 controls with DM2 and 25 healthy controls). All 80 participants with a DXA-scan were included in the present study. This was a part of a larger study and the present study was designed as an exploratory study. Thus, a power calculation was not done for outcomes presented in this study.

Inclusion criteria for the present study was participation in the study "HIV-infected persons with type 2 diabetes show evidence of endothelial dysfunction and increased inflammation" [16] and willingness to participate with a DXA scan. For the study "HIV-infected persons with type 2 diabetes show evidence of endothelial dysfunction and increased inflammation" the following inclusion criteria were used: Inclusion criteria for PLWH were treatment with cART and undetectable viral replication (defined as HIV RNA $<200$ copies/mL). Inclusion criteria for persons with DM2 were confirmed DM2 by a trained clinician and one or more of the following: $\mathrm{HbA} 1 \mathrm{c} \geq 48 \mathrm{mmol} / \mathrm{mol}$, fasting venous plasma glucose $>$ $7 \mathrm{mmol} / \mathrm{l}$, or 2 -h venous plasma glucose concentration on $\geq 11.1 \mathrm{mmol} / \mathrm{L}$ after a glucose tolerance test prior to this study $[17,18]$. All persons with DM2 were treated with diet and/or oral anti-diabetics and/or insulin. All persons without DM2 were required to have both normal fasting venous plasma glucose $(<6.1 \mathrm{mmol} / \mathrm{L})$ and $\mathrm{HbA} 1 \mathrm{c}<48 \mathrm{mmol} / \mathrm{mol}$. We did not have to exclude any participants without DM2 included in the study due to elevated plasma glucose level or HbA1c.

Exclusion criteria were immunosuppressive treatment, acute infections, malignancy, and pregnancy. However, no females in the reproductive age group were included in the study. Both written and oral information was given to the study participants prior to inclusion in the study. This included instructions about at least 10-h fasting before time of blood sampling.

All PLWH with DM2 attending routine control for HIV infection at the Department of Infectious Diseases at University Hospital of Copenhagen, Rigshospitalet or Hvidovre Hospital, and who fulfilled inclusion and exclusion criteria for the study "HIV-infected persons with type 2 diabetes show evidence of endothelial dysfunction and increased inflammation" [16], were invited to participate until $n=25$ were recruited in this group. Inclusion of patients and controls in the other groups was done to achieve best possible match on age and sex. Persons with DM2 were included from the Department of Endocrinology and Centre of Inflammation and Metabolism, University Hospital of Copenhagen, Rigshospitalet. Healthy controls were recruited among hospital staff. All PLWH included in the study had a confirmatory 
positive HIV test. A negative HIV test was not performed for participants in the uninfected control groups, since the prevalence of HIV in Denmark is $0.1 \%$, and it seems reasonable to assume that clinically healthy persons are HIV-negative. Six controls with DM2 also participated in a study concerning the effect of short duration, high-intensity interval training on endothelial function and metabolism [19]. These participants were included before training.

The study was performed in accordance with the Declaration of Helsinki and approved by the local ethical committee on health research ethics (H-4-2012-076 CIM VEK) and the Danish Data Protection Agency.

\section{Dual-energy X-ray absorptiometry scan}

A DXA-scan was used to measure total fat mass, limb fat mass, and trunk fat mass in all participants (Lunar Prodigy Advance; GE Medical Systems Lunar, Milwaukee, WI, USA). Prodigy Software (enCORE 2004, version 8.8, GE Lunar Corp., Madison, WI, USA).

\section{Laboratory analyses}

Fasting venous blood samples were collected from all participants. HIV RNA was measured in PLWH, and glucose, HbA1c, triglyceride, cholesterol, HDL, LDL, cpeptide, CD4+, and CD8+ counts were measured in all participants as routine analyses at the time of inclusion at the Department of Clinical Biochemistry at Rigshospitalet, Denmark. Tubes with sodium fluoride and potassium oxalate were used to obtain plasma for glucose analyses and tubes with lithium heparin separator were used to obtain plasma for triglyceride, cholesterol, HDL, LDL an c-peptide analyses. The samples were centrifuged within $4 \mathrm{~h}$. Tubes with $\mathrm{K}_{2}$ EDTA were used to collect full blood for HbA1c analyses. All tubes were from Greiner Bio-One, Kremsmünster, Austria.

Concentrations of plasma adiponectin, IL- 6 and TNF$\alpha$ were measured in snap-frozen plasma. Adiponectin was measured using sandwich immunoassay, Human adiponectin Kit (MSD, Gaithersburg, MD, USA). IL-6 and TNF- $\alpha$ were measured using a sandwich immunoassay, Proinflammatory Panel 1 (MSD, Rockville, MD, USA). sCD14 was measured in snap-frozen plasma using an enzyme-linked immunosorbent assay ( $R \& D$, Minneapolis, USA). All assays were performed according to manufacturers' instructions.

\section{Insulin resistance}

To measure insulin resistance, we used the Homeostatic Model Assessment of Insulin Resistance (HOMA-IR) [20]. HOMA-IR was calculated using HOMA Calculator (https://www.dtu.ox.ac.uk/ToolsSoftware/) including fasting glucose and C-peptide.

\section{Statistics}

Data were tested for normal distribution, and all soluble markers were logarithmic transformed to obtain normal distribution. Results are given as mean and 95\% Confidence Interval $(95 \% \mathrm{CI})$ or geometric mean (95\% CI). Differences between groups were analyzed using oneway ANOVA followed by $\mathrm{t}$ test. Main outcomes and possible interactions between HIV infection and DM2 were further investigated using multivariate linear regression. The soluble markers: adiponectin, IL-6, and TNF- $\alpha$ were correlated to fat mass in $\mathrm{kg}$ to assess their correlation to fat distribution. Only significant correlations were reported. Correlations were done using Pearson correlation. Pearson chi-square test was used on categorical data. Two-tailed $p$-values $<0.05$ were considered significant Statistical analyses were performed using SPSS version 25 (SPSS, Inc.; Chicago, IL, USA), GraphPad Prism 5 (GraphPad Software, San Diego, CA, USA) and $\mathrm{R}$ version 3.5.2.

\section{Results \\ Study population}

Characteristics of the study population are shown in Table 1. The groups were similar regarding age and sex. There was no difference in treatment duration between the two PLWH groups. HOMA-IR was comparable in $\mathrm{HIV}+\mathrm{DM} 2+$ and controls with DM2 but elevated compared to HIV + DM2- and healthy controls.

\section{Fat distribution}

HIV + DM2+ had a non-statistically significant tendency towards more total fat measured in $\mathrm{kg}$ and percentages compared to HIV + DM2- $(p=0.059$ and $p=0.073$, respectively) and towards more trunk fat mass $(p=0.057$ and $p=0.063$, respectively) (Table 2 ).

No difference in limb fat mass measured in $\mathrm{kg}$ and percentages was found between HIV + DM2+ and HIV + DM2- $(p=0.130$ and $p=0.111)$. Furthermore, no difference in trunk/ limb fat ratio was found between HIV + DM2+ and HIV + DM2- $(p=0.444)$ (Table 2).

We found comparable total fat mass, trunk fat mass and limb fat mass when measured in $\mathrm{kg}$ in HIV + DM2+ and controls with DM2 $(p=0.495, p=0.254$, and $p=$ 0.068 , respectively). However, HIV + DM2+ had low percentages of total fat mass, trunk fat mass and limb fat mass than controls with $\operatorname{DM} 2(p=0.038, p=0.042$ and $p=0.054$, respectively) without however reaching statistical significance in the latter. Also, HIV + DM2+ had a higher trunk /limb fat ratio than controls with DM2 $(p=0.013)$ (Table 2).

HIV + DM2+ and the healthy controls had comparable total fat mass measured in $\mathrm{kg}$ and percentages $(p=0.157$ and $p=0.887$, respectively). However, HIV $+\mathrm{DM} 2+$ had more trunk fat mass than the healthy controls when 
Table 1 Clinical characteristics of the study population

\begin{tabular}{|c|c|c|c|c|c|}
\hline & HIV + DM2+ & HIV + DM2- & Controls with DM2 & Healthy controls & $P$ \\
\hline$n$ & 18 & 18 & 19 & 25 & \\
\hline Age, median (range) & $58(46-65)$ & $57(38-67)$ & $57(49-66)$ & $58(42-66)$ & 0.801 \\
\hline Gender (\%male) & 89 & 94 & 68 & 88 & 0.125 \\
\hline BMI & $27(25-29)$ & $25(22-27)$ & $29(27-30)$ & $25(24-26)$ & 0.003 \\
\hline \multicolumn{6}{|l|}{ Metabolic factors } \\
\hline Fasting -PG & $8.6(7.2-10.0)$ & $5.2(5.0-5.4)^{*}$ & $8.5(7.5-9.6)$ & $5.3(5.1-5.5)^{*}$ & $<0.001$ \\
\hline Hba1c (mmol/mol) & $49(44-55)$ & $35(33-37)^{*}$ & $55(49-60)$ & $37(36-38)^{*}$ & $<0.001$ \\
\hline Cholesterol & $4.5(4.1-4.9)$ & $5.6(5.0-6.1)^{*}$ & $4.2(3.8-4.7)$ & $5.5(5.1-5.9)^{*}$ & $<0.001$ \\
\hline $\mathrm{HDL}$ & $1.1(0.9-1.4)$ & $1.4(1.2-1.6)$ & $1.3(1.1-1.4)$ & $1.5(1.3-1.7)^{*}$ & 0.010 \\
\hline LDL & $2.3(1.9-2.7)$ & $3.4(2.8-4.0)^{*}$ & $2.4(1.9-2.8)$ & $3.5(3.2-3.8)^{*}$ & $<0.001$ \\
\hline Triglyceride & $2.7(1.8-3.7)$ & $2.0(1.2-2.8)$ & $2.0(1.5-2.5)$ & $1.4(1.0-2.0)^{*}$ & 0.006 \\
\hline HOMA-IR & $3.3(2.6-3.9)$ & $2.0(1.6-2.3)^{*}$ & $3.3(2.5-4.1)$ & $1.7(1.4-2.0)^{*}$ & $<0.001$ \\
\hline Fasting c-peptide, median (range) & $0.96(0.58-1.44)$ & $1.09(0.66-2.18)^{*}$ & $1.05(0.39-2.32)$ & $0.75(0.39-1.42)^{*}$ & $<0.001$ \\
\hline \multicolumn{6}{|l|}{ Immunological factors } \\
\hline Time since HIV diagnosis (months) & $168(116-219)$ & $205(147-263)$ & - & - & 0.413 \\
\hline CD4 count & $689(537-842)$ & $656(506-807)$ & $1197(986-1408)^{*}$ & $822(711-932)$ & $<0.001$ \\
\hline CD8 count & $885(748-1023)$ & $1003(737-1270)$ & $485(436-735)^{*}$ & $446(364-528)^{*}$ & $<0.001$ \\
\hline Nadir CD4 & $188(114-262)$ & $219(118-320)$ & - & - & 0.608 \\
\hline HIV RNA (copies/mL) & $28(15-42)$ & $29(16-43)$ & - & - & 0.845 \\
\hline \multicolumn{6}{|l|}{ Medication (current) } \\
\hline HIV treatment duration (months) & $130(97-164)$ & $125(87-164)$ & - & - & 0.835 \\
\hline Lipid lowering drug (\%) & 72 & 6 & 58 & 16 & $<0.001$ \\
\hline Insulin (\%) & 24 & - & 6 & - & 0.129 \\
\hline Metformin (\%) & 67 & - & 68 & - & 0.909 \\
\hline GLP-1 (\%) & 6 & - & 26 & - & 0.087 \\
\hline DPP-4 (\%) & 11 & - & 11 & - & 0.954 \\
\hline Sulphonylureas (\%) & 28 & - & 21 & - & 0.634 \\
\hline Special diet (\%) & 6 & - & 21 & - & 0.168 \\
\hline
\end{tabular}

Differences between groups were analyzed using one-way ANOVA followed by t-test for comparison between HIV + DM2+ and the 3 control groups when ANOVA test was $<0.05 .{ }^{*} p \leq 0.05$ compared to HIV + DM2+. For categorical data Pearson chi-square test was used. Results are given as mean $(95 \% \mathrm{Cl})$ if not otherwise stated. Participants could receive more than one type of drug for diabetes treatment and can be included in more than one treatment category except in the diet group. Participants were only registered as diet treated if this was the only treatment they received for their type 2 diabetes. Abbreviations: HIV +DM2+ Persons living with HIV with diabetes mellitus type 2, HIV + DM2- Persons living with HIV without diabetes mellitus type 2, BMI Body mass index, Fasting-PG Fasting plasma glucose, Hba1c Hemoglobin A1c, HDL High density lipoprotein, LDL Low density lipoprotein, HOMA-IR Homeostatic Model Assessment of Insulin Resistance

Table 2 Fat distribution

\begin{tabular}{|c|c|c|c|c|c|}
\hline & HIV + DM2+ & HIV + DM2- & Controls with DM2 & Healthy controls & $P$ \\
\hline Total fat (kg) & $25.5(19.8-31.0)$ & $18.8(14.1-23.4)$ & $28.9(25.8-32.0)$ & $21.6(19.3-24.0)$ & 0.002 \\
\hline Trunk fat (kg) & $17.2(13.5-20.9)$ & $12.4(8.9-15.9)$ & $18.6(16.2-21.0)$ & $13.0(11.5-14.6)^{*}$ & 0.002 \\
\hline Limb fat (kg) & $7.5(5.5-9.5)$ & $5.7(4.1-7.2)$ & $9.5(8.4-10.5)$ & $7.9(6.7-9.1)$ & 0.004 \\
\hline Total fat (\%) & $28.8(24.4-33.3)$ & 23.4(19.1-27.7) & $34.0(31.5-36.6)^{*}$ & $28.5(25.8-31.2)$ & 0.001 \\
\hline Trunk fat (\%) & $34.3(29.7-38.8)$ & $28.1(23.0-33.2)$ & $39.4(36.8-42.0)^{*}$ & $33.5(30.9-36.2)$ & 0.001 \\
\hline Limb fat (\%) & $46.6(36.3-57.0)$ & $36.5(28.6-44.5)$ & $58.0(51.6-64.5)$ & $47.6(41.4-53.8)$ & 0.002 \\
\hline Trunk fat mass/limb fat mass ratio & $2.58(2.18-2.97)$ & $2.34(1.84-2.85)$ & $2.01(1.77-2.26)^{*}$ & $1.74(1.55-1.93)^{*}$ & 0.001 \\
\hline
\end{tabular}

Differences between groups were analyzed using one-way ANOVA followed by t-test for comparison between HIV + DM2+ and the 3 control groups. * $p \leq 0.05$ compared to HIV + DM2+. Abbreviations: HIV + DM2+ Persons living with HIV with diabetes mellitus type 2, HIV + DM2- Persons living with HIV without diabetes mellitus type 2 
measured in $\mathrm{kg}$ but not in percentages $(p=0.020$ and $p=0.746$, respectively). No difference in limb fat mass was found between the two groups when measured in $\mathrm{kg}$ and percentages $(p=0.685$ and $p=0.855$, respectively). HIV + DM2+ had a higher trunk/limb fat ratio when compared to healthy controls $(p<0.001)$ (Table 2 and Fig. 1.a).

\section{Adiponectin}

$\mathrm{HIV}+\mathrm{DM} 2+$ had lower plasma adiponectin than HIV + DM2- and healthy controls $(p=0.037$ and $p=0.001$, respectively). In contrast, $\mathrm{HIV}+\mathrm{DM} 2+$ had comparable concentration of plasma adiponectin to controls with DM2 ( $p=0.345)$ (Table 3).

In the total population plasma adiponectin was negatively correlated with both trunk fat mass $(\mathrm{kg})$ and trunk fat/ limb fat ratio $(r=-0.301, p=0.007$ and $r=-0.541$, $p<0.001$, respectively). Furthermore, plasma adiponectin was negatively correlated with HOMA-IR $(r=-0.464$, $p<0.001)$.

\section{IL-6 and TNF-a}

$\mathrm{HIV}+\mathrm{DM} 2+$ had higher concentration of IL-6 compared to HIV + DM2- and healthy controls $(p=0.039$

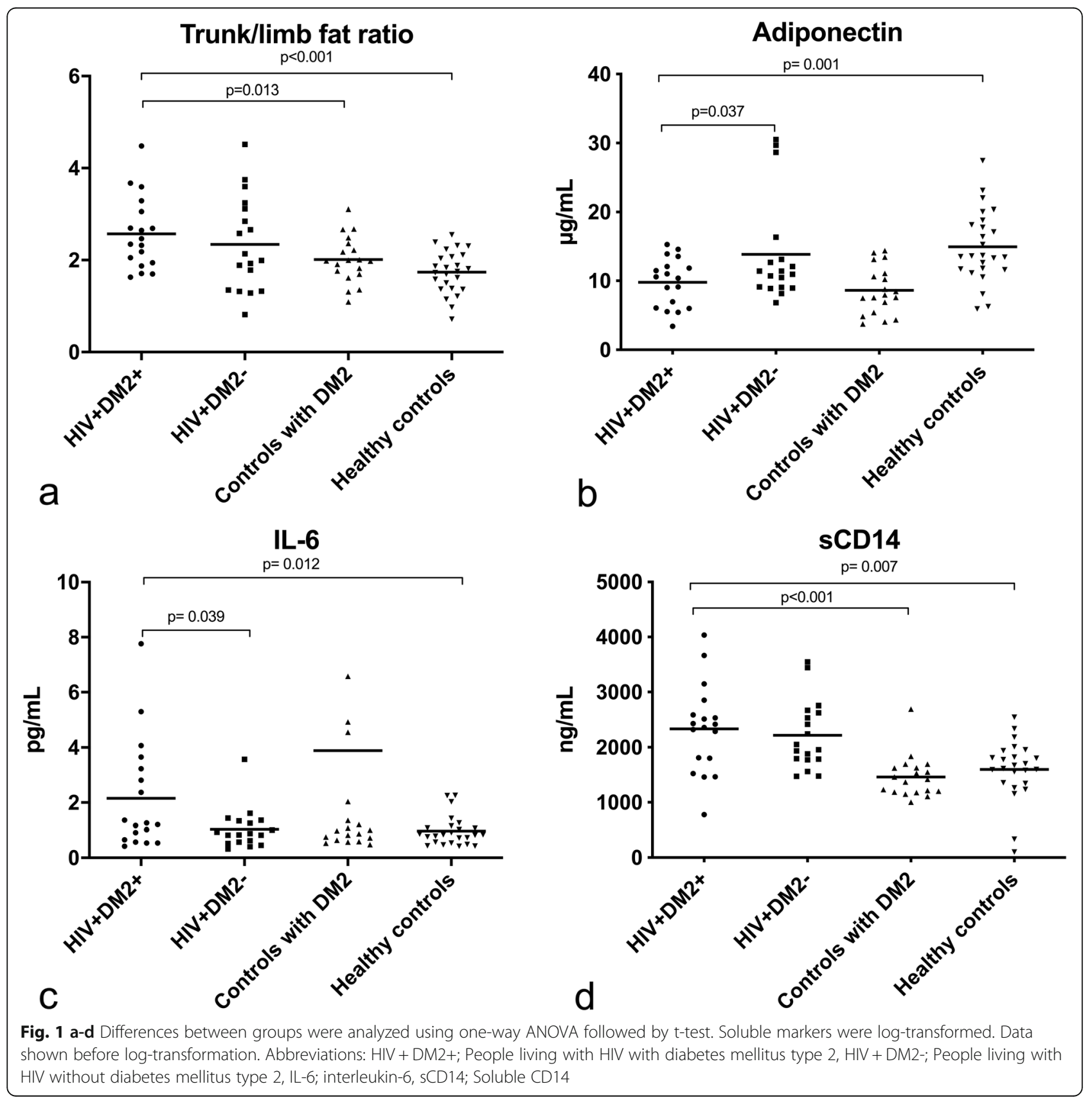


Table 3 Soluble markers

\begin{tabular}{llllll}
\hline & HIV + DM2+ & HIV + DM2- & Controls with DM2 & Healthy controls & $\boldsymbol{P}$ \\
\hline Adiponectin $(\mu \mathrm{g} / \mathrm{mL})$ & $9.79(8.02-11.56)$ & $13.83(10.07-17.06)^{*}$ & $8.63(6.96-10.29)$ & $14.96(12.80-17.11)^{*}$ & $<0.001$ \\
IL-6 $(\mathrm{pg} / \mathrm{mL})$ & $2.16(1.16-3.15)$ & $1.04(0.67-1.41)^{*}$ & $3.89(0.89-8.66)$ & $0.91(0.72-1.11)^{*}$ & 0.050 \\
TNF-alfa $(\mathrm{pg} / \mathrm{mL})$ & $3.24(2.66-3.82)$ & $2.92(2.01-3.84)$ & $4.02(1.32-6.73)$ & $2.29(2.07-2.51)$ & 0.112 \\
sCD14 $(\mathrm{ng} / \mathrm{mL})$ & $2333(1935-2732)$ & $2218(1912-2525)$ & $1461(1277-1644)^{*}$ & $1504(1302-1706)^{*}$ & $<0.001$ \\
\hline
\end{tabular}

Soluble markers presented as mean $(95 \% \mathrm{Cl})$ before log transformation. P-value represent one-way ANOVA analyses after log transformation followed by t-test for comparison between HIV + DM2+ and the 3 control groups when ANOVA test was * $p<0.05$ compared to HIV+DM2+

Abbreviations: HIV + DM2+ People living with HIV with diabetes mellitus type 2, HIV + DM2- People living with HIV without diabetes mellitus type 2, IL-6 Interleukin-6, TNF- a Tumor necrosis factor-alfa, sCD14 Soluble CD14, DM2 Diabetes mellitus type 2

and $p=0.012$, respectively). However, IL- 6 concentration was comparable in HIV + DM2+ and controls with DM2 $(p=0.825)$. Furthermore, in the total population IL-6 was positively correlated with both trunk fat mass and HOMA-IR $(r=0.253, p=0.024$ and $r=0.287, p=0.013$, respectively).

No difference in TNF- $\alpha$ was found between the groups (Table 3).

\section{SCD14}

$\mathrm{HIV}+\mathrm{DM} 2+$ and HIV + DM2- had comparable concentration of $\operatorname{sCD} 14(p=0.850)$. However, HIV + DM2+ had higher concentration of sCD14 compared to both controls with DM2 and healthy controls $(p<0.001$ and $p=$ 0.007 , respectively) (Table 3 ).

\section{HIV status, DM2 status and association to fat distribution} and soluble markers

To adjust for potential confounders and formally test for interactions between HIV infection and DM2 we performed a multiple linear regression model including HIV status, DM2 status, age and sex. Furthermore, BMI was included when one of the soluble markers were used as outcome (Tables 4 and 5).

\section{Fat distribution}

In the unadjusted model HIV infection was associated with less limb fat mass $(p=0.005)$ and with higher trunk/limb fat ratio $(p<0.001)$. This was consistent after adjusting ( $p=0.008$ and $p=0.002$, respectively).

In the unadjusted model DM2 was independently associated with more total fat mass $(\mathrm{kg})(p<0.001)$, trunk fat mass $(\mathrm{kg})(p<0.001)$ and limb fat mass $(\mathrm{kg})(p=$ $0.037)$. This was consistent after adjusting $(p<0.001$, $p<0.001$ and $p=0.045$, respectively) (Table 4).

\section{Soluble markers}

In the unadjusted model HIV infection was associated with higher concentration of $\operatorname{sCD} 14(p<0.001)$ which was consistent after adjusting $(p<0.001)$. HIV infection was not associated with TNF- $\alpha$ in the unadjusted model but after adjusting HIV infection was associated with TNF- $\alpha(p=0.018)$.
In the unadjusted model DM2 was associated with lower concentration of plasma adiponectin $(p<0.001)$ and higher concentration of IL-6 $(p=0.005)$ and TNF- $\alpha$ $(p=0.033)$. After adjusting DM2 was still associated with lower concentration of plasma adiponectin $(p<0.001)$ and higher concentration of IL-6 ( $p=0.020)$ (Table 5).

\section{Interactions}

No interactions were found between HIV infection and DM2 in an unadjusted model for any of the depending variables (Tables 4 and 5).

\section{Discussion}

We found no evidence of a synergistic effect of HIV infection and DM2 on fat distribution or inflammatory markers in this study. Both PLWH with DM2 and controls with DM2 had low plasma adiponectin and high IL-6 concentrations. Furthermore, PLWH had higher sCD14 concentrations and high trunk/limb fat ratio compared to controls with DM2 and healthy controls.

In this study PLWH both with and without DM2 had higher trunk/limb fat ratio compared to controls with DM2 and healthy controls. Elevated trunk/limb fat ratio is often included in the clinical diagnosis of lipodystrophy in PLWH and is associated with increased risk of CVD [21, 22]. Altered fat distribution has been related to exposure to older generation cART [5]. Since PLWH included in the study had been living with HIV for median 14 years, the altered fat ratio could be related to exposure to older generation of cART.

Adiponectin is an adipokine with anti-inflammatory properties. Reduction in plasma adiponectin levels has been associated with increased CVD risk in both PLWH and uninfected controls. As reported in this systematic review and meta-analyses previous studies described lower concentration of plasma adiponectin in persons with DM2 [10]. Accordingly, lower concentration of plasma adiponectin was found in HIV + DM2+ than in HIV + DM2-. Furthermore, no difference in plasma adiponectin concentration between $\mathrm{HIV}+\mathrm{DM} 2+$ and controls with DM2 and plasma adiponectin was negatively correlated with HOMA-IR in this cohort. Adiponectin is produced by adipocytes and is decreased in obesity due 


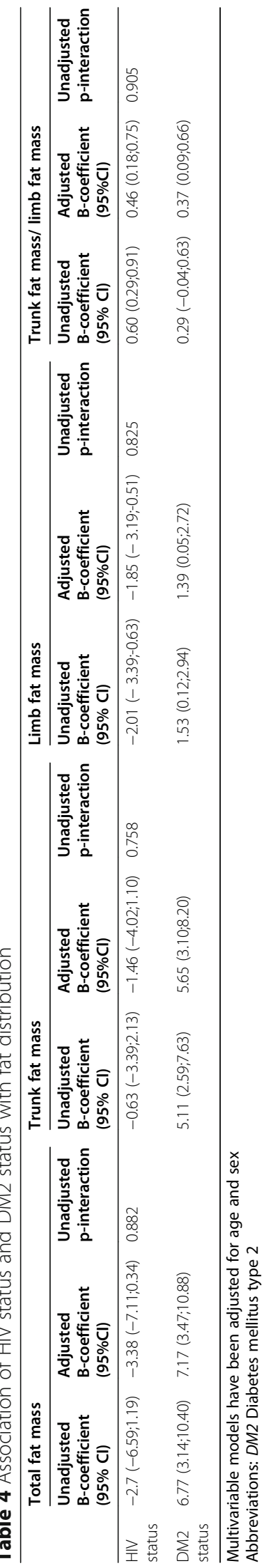




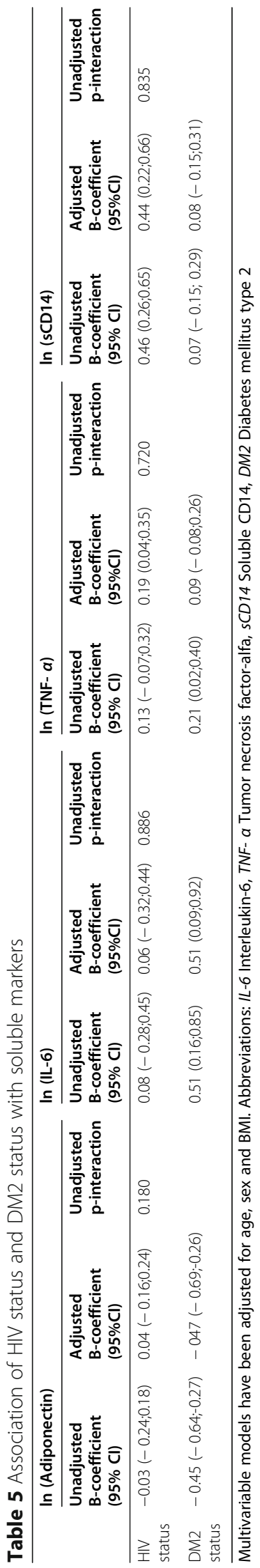


to dysfunctional hypertrophic adipocytes [23]. Especially, visceral fat accumulation has been associated with lower plasma adiponectin in both PLWH and in the general population which is consistent with the negative correlation between trunk fat mass and plasma adiponectin found in this study $[9,23]$. HIV + DM2+ and controls with DM2 had comparable amount of trunk fat mass measured in $\mathrm{kg}$, which may explain the similar concentrations of plasma adiponectin in the two groups.

As in adiponectin production, adipose tissue plays an important role in the production of IL-6 [23] and several studies have shown that obesity is associated with elevated IL- 6 concentration in both PLWH as well as in the general population $[15,24]$. We did hypothesize a synergistic effect of HIV infection and DM2 on IL-6 concentrations since HIV infection has also been shown to induce IL-6 production [25] and higher concentration of IL- 6 are found in PLWH compared to non-infected controls $[14,24]$. Contrary to our hypotheses IL-6 concentration was comparable in HIV + DM2+ and controls with DM2 and positively correlated with both HOMAIR and trunk fat mass. We speculated that in the context of well-treated and virally suppressed PLWH, metabolicdriven inflammation may outweigh pro-inflammatory stimuli associated with chronic HIV infection. Also, different use of medication with anti-inflammatory properties between the groups could have affected our results. More HIV + DM2+ were treated with lipid lowering drugs including statins than the participants in the other groups. Besides the lipid-lowering effect statins are thought to have anti-inflammatory properties since statin use is found to reduce CRP levels and perhaps increase adiponectin in the general population [26, 27]. However, smaller studies investigating the impact of statin use on inflammatory markers in PLWH could not confirm this effect in PLWH $[28,29]$. Furthermore, more controls with DM2 received glucagon-like-peptide1 (GLP-1) analogues, although not significantly, which is also thought to have anti-inflammatory properties [30]. Thus, differences in statin and GLP-1 use between the groups may be a confounder in this study. However, due to the sample size we were not able to adjust for this in our analyses. Importantly, both low plasma adiponectin and elevated IL-6 are associated with increased risk of CVD in both PLWH and the general population [11, 15, 31, 32].

We did not find any difference in TNF- $\alpha$ concentrations between the groups. However, higher TNF- $\alpha$ concentration was associated with HIV infection in the adjusted analysis which is consistent with previous studies [13, 33].

sCD14 is a marker of monocyte activation [34] and elevated concentrations are reported in PLWH [35]. Furthermore, elevated concentration of SCD14 have been associated with increased mortality in PLWH [36].
Importantly, DM2 has been associated with microbial translocation and elevated concentrations of lipopolysaccharide (LPS), which is a strong inducer of sCD14 [37]. In the present study, we found elevated concentrations of sCD14 in PLWH both with and without DM2 compared to both controls with DM2 and healthy controls. A larger study found decreased concentration of sCD14 in HIV/obese persons compared to HIV/non-obese persons [38]. In contrast, an increase in sCD14 concentration in overweight and obese PLWH gaining weight after initiation of cART has been reported [39]. To our knowledge data on $\mathrm{SCD} 14$ and DM2 is still very limited both in PLWH and uninfected persons. Our data suggest that elevated concentration of SCD14 is related to HIV status and not DM2 or fat distribution.

This study is limited by its cross-sectional design and relatively small number of participants. Furthermore, it is a limitation that HIV testing was not performed consistently in all participants resulting in a risk of undiagnosed HIV in the control groups. However, the prevalence of HIV in Denmark is low $(0.1 \%)$, and it seems unlikely that undiagnosed HIV is a major confounder. Also, more detailed information concerning complications to DM2 would have added valuable information to the clinical characteristics of the study population. However, the study included three control groups; PLWH without DM2, persons with DM2 and healthy controls matched on age allowing us to determine association of both HIV infection and DM2 and is to the best of our knowledge the first study to investigate the combined effect of HIV infection and DM2 on fat distribution and inflammation in this setting.

\section{Conclusion}

In conclusion, both well-treated PLWH in the contemporary cART era and persons with DM2 had altered fat distribution and chronic inflammation. However, no evidence of a synergistic effect of HIV infection and DM2 on fat distribution, plasma adiponectin or inflammatory markers was found. However, our data suggest that HIV is associated with higher trunk/limb fat ratio, higher concentration of sCD14 and possible higher concentration of TNF- $\alpha$. Furthermore, DM2 was associated with increased trunk fat mass, low concentration of plasma adiponectin, and high concentration of IL-6, probably driven in part by increased trunk fat mass. Thus, persons living with both diseases may have a higher inflammatory state driven by both fat tissue and HIV induced immune activation. This was an exploratory study with a limited power, but it points to markers that should be further investigated to gain knowledge about the mechanisms and risk factors related to the increased risk of CVD in PLWH with DM2. 


\section{Abbreviations}

CVD: Cardiovascular disease; CART: Combination antiretroviral treatment; DM2: Diabetes mellitus type 2; DXA-scan: Dual-energy X-ray absorptiometry scan; HOMA-IR: Homeostatic Model Assessment of Insulin Resistance (HOMAIR); IL-6: Interleukin-6; PLWH: Persons living with HIV; HIV + DM2+: Persons living with HIV with DM2; HIV + DM2-: Persons living with HIV without DM2; sCD14: Soluble CD14; TNF- $a$ : Tumor necrosis factor-alfa

\section{Acknowledgements}

Not applicable.

\section{Authors' contributions}

MHS, MT, JCG, BL and SDN designed the study. MHS, JA and LK included the patients. MHS, SDN, JA and JCG were involved in data collection. MHS, MT, $\mathrm{BL}$ and $\mathrm{HU}$ were involved in the laboratory analyses. JA performed the DXA-scans. MHS, MG and SDN were responsible for the statistical analyses. All authors interpreted the data. MHS drafted the manuscript. All authors have critically revised and approved the final version.

\section{Funding}

The study was supported with grants from Novo Nordisk Foundation, Rigshospitalet University Hospital of Copenhagen, and the Augustinus Foundation. The funders had no role in the study design, data collection or analysis, preparation of the manuscript or decision to submit.

\section{Availability of data and materials}

The dataset analysed during the current study is available from the corresponding author on reasonable request.

\section{Ethics approval and consent to participate}

The study was performed in accordance with the Declaration of Helsinki and approved by the local ethical committee on health research ethics, "Videnskabsetisk Komitee, Region Hovedstaden" which belongs to The Danish National Committee on Health Research Ethics (H-4-2012-076 CIM VEK) and the Danish Data Protection Agency. All participants signed a written consent when included in the study.

\section{Consent for publication}

Not applicable.

\section{Competing interests}

MG: unrestricted travel grants from Gilead; SDN: Unrestricted research grants from Novo Nordisk Foundation, Lundbeck Foundation, Augustinus Foundation, Rigshospitalet Research Council. Travelling grants from Gilead and GSKNiiV. Advisory board activity for Gilead and GSKNiiV. All other authors have no competing interests. Part of the data were presented as a poster presentation at Conference on Retroviruses and Opportunistic Infections (CROI), Seattle, February 23 to February 26, 2015.

\section{Author details}

'Department of Infectious Diseases, Rigshospitalet, Copenhagen University Hospital, Copenhagen, Denmark. ${ }^{2}$ The Centre of Inflammation and Metabolism and the Centre for Physical Activity Research, Rigshospitalet, University of Copenhagen, Copenhagen, Denmark. ${ }^{3}$ Department of Infectious Diseases, Hvidovre Hospital, University of Copenhagen, Hvidovre, Denmark. ${ }^{4}$ Department of Pulmonary- and Infectious Diseases, Nordsjællands Hospital, University of Copenhagen, Hillerød, Denmark. ${ }^{5}$ Department of Clinical Immunology, Rigshospitalet, Copenhagen University Hospital, Copenhagen, Denmark. 'Section of Clinical Immunology and Infectious Diseases, Oslo University Hospital, Oslo, Norway. ${ }^{7}$ Research Institute of Internal Medicine, Oslo University Hospital, Oslo, Norway. ${ }^{8}$ Institute for Clinical Medicine, University of Oslo, Oslo, Norway.

\section{Received: 15 October 2019 Accepted: 5 November 2020}

\section{Published online: 25 November 2020}

\section{References}

1. The Antiretroviral Therapy Cohort Collaboration. Causes of death in HIV-1infected patients treated with antiretroviral therapy, 1996-2006: collaborative analysis of 13 HIV cohort studies. Clin Infect Dis. 2010;50:138796. https://doi.org/10.1086/652283.
2. Legarth RA, Ahlström MG, Kronborg G, Larsen CS, Pedersen C, Pedersen $\mathrm{G}$, et al. Long-term mortality in HIV-infected individuals 50 years or older: a Nationwide, population-based cohort study. Jaids. 2016;71:2138. https://doi.org/10.1097/QAl.0000000000000825.

3. Freiberg MS, Chang C-CH, Kuller LH, Skanderson M, Lowy E, Kraemer KL, et al. HIV infection and the risk of acute myocardial infarction. JAMA Intern Med. 2013;173:614-22. https://doi.org/10.1001/jamainternmed.2013.3728.

4. Islam FM, Wu J, Jansson J, Wilson DP. Relative risk of cardiovascular disease among people living with HIV: a systematic review and meta-analysis. HIV Med. 2012;13:453-68.

5. Gelpi M, Afzal S, Fuchs A, Lundgren J, Knudsen AD, Drivsholm N, et al. Prior exposure to thymidine analogs and didanosine is associated with longlasting alterations in adipose tissue distribution and cardiovascular risk factors. AIDS. 2019;33:675-83.

6. Hasse B, Iff M, Ledergerber B, Calmy A, Schmid P, Hauser C, et al. Obesity trends and body mass index changes after starting antiretroviral treatment: the Swiss HIV cohort study. Open forum Infect Dis. 2014;1:ofu040. https:// doi.org/10.1093/ofid/ofu040.

7. Herrin M, Connecticut BAVA, System H, Haven W, Tate JP, Va SD, et al. Weight gain and incident diabetes among HIV infected-veterans initiating antiretroviral therapy compared to uninfected individuals. JAIDS J Acquir Immune Defic Syndr. 2016;73:228-36.

8. Achhra A, Mocroft A, Reiss P, Sabin C, Ryom L, de Wit S, et al. Short-term weight gain after antiretroviral therapy initiation and subsequent risk of cardiovascular disease and diabetes: the D:a: D study. HIV Med. 2015;17(4): 255. https://doi.org/10.1111/hiv.12294.

9. Freitas P, Carvalho D, Santos AC, Madureira AJ, Martinez E, Pereira J, et al. Adipokines, hormones related to body composition, and insulin resistance in HIV fat redistribution syndrome. BMC Infect Dis. 2014;14:347. https://doi. org/10.1186/1471-2334-14-347.

10. Li S, Shin HJ, Ding EL, van Dam RM. Adiponectin levels and risk of type 2 diabetes: a systematic review and meta-analysis. JAMA. 2009;302:179-88.

11. Ketlogetswe KS, Post WS, Li X, Palella FJ, Jacobson LP, Margolick JB, et al. Lower adiponectin is associated with subclinical cardiovascular disease among HIV-infected men. AIDS. 2014;28:901-9. https://doi.org/10.1097/QAD. 0000000000000186.

12. Schulze MB, Shai I, Rimm EB, Li T, Rifai N, Hu FB. Adiponectin and future coronary heart disease events among men with type 2 diabetes. Diabetes. 2005;54:534-9. https://doi.org/10.2337/diabetes.54.2.534.

13. Wada NI, Jacobson LP, Margolick JB, Breen EC, Macatangay B, Penugonda S, et al. The effect of HAART-induced HIV suppression on circulating markers of inflammation and immune activation. AIDS. 2015;29:463-71.

14. Neuhaus J, Jacobs DR, Baker JV, Calmy A, Duprez D, La Rosa A, et al. Markers of inflammation, coagulation, and renal function are elevated in adults with HIV infection. J Infect Dis. 2010;201:1788-95. https://doi.org/10.1086/652749.

15. Donath MY, Shoelson SE. Type 2 diabetes as an inflammatory disease. Nat Rev Immunol. 2011;11:98-107. https://doi.org/10.1038/nri2925.

16. Hove-Skovsgaard M, Gaardbo JC, Kolte L, Winding K, Seljeflot I, Svardal A, et al. HIV-infected persons with type 2 diabetes show evidence of endothelial dysfunction and increased inflammation. BMC Infect Dis. 2017;17:1-8.

17. Geneva: World Health Organization. Defintion and diagnosis diabetes Mellistus and lintermediate hyperglycemia; 2006.

18. Use of Glycated Haemoglobin ( $\mathrm{HbA1c}$ ) in the Diagnosis of Diabetes Mellitus: Abbreviated Report of a WHO Consultation. 2011. http://www.ncbi. nlm.nih.gov/pubmed/26158184.

19. Winding KM, Munch GW, lepsen UW, Van Hall G, Pedersen BK, Mortensen SP. The effect on glycaemic control of low-volume high-intensity interval training versus endurance training in individuals with type 2 diabetes. Diabetes Obes Metab. 2018;20:1131-9.

20. Wallace TM, Levy JC, Matthews DR. Use and abuse of HOMA modeling. Diabetes Care. 2004;27:1487-95 http://www.ncbi.nlm.nih.gov/pubmed/15161807.

21. Carr A, Emery S, Law M, Puls R, Lundgren JD, Powderly WG, et al. An objective case definition of lipodystrophy in HIV-infected adults: a case-control study. Lancet. 2003;361:726-35 http://ovidsp.ovid.com/ ovidweb.cgi?T=JS\&PAGE=reference $\& D=$ emed6\&NEWS=N\&AN=2003105 925.

22. Lake JE, Wohl D, Scherzer R, Grunfeld C, Tien PC, Sidney S, et al. Regional fat deposition and cardiovascular risk in HIV infection: the FRAM study. AIDS Care. 2011;23:929-38. https://doi.org/10.1080/09540121.2010.543885.

23. Hajer GR, Van Haeften TW, Visseren FLJ. Adipose tissue dysfunction in obesity, diabetes, and vascular diseases. Eur Heart J. 2008;29:2959-71. 
24. Conley LJ, Bush TJ, Rupert AW, Sereti I, Patel P, Brooks JT, et al. Obesity is associated with greater inflammation and monocyte activation among HIVinfected adults receiving antiretroviral therapy. Aids. 2015;29:2201-7.

25. Breen EC, Rezai AR, Nakajima K, Beall GN, Mitsuyasu RT, Hirano T, et al. Infection with HIV is associated with elevated IL-6 levels and production. J Immunol. 1990;144:480-4 http://www.ncbi.nlm.nih.gov/pubmed/2295799.

26. Albert MA, Danielson E, Rifai N, Ridker PM, for the PRINCE Investigators. Effect of statin therapy on C-reactive protein levels. JAMA. 2001;286:64. https://doi.org/10.1001/jama.286.1.64.

27. Chruściel P, Sahebkar A, Rembek-Wieliczko M, Serban MC, Ursoniu S, Mikhailidis DP, et al. Impact of statin therapy on plasma adiponectin concentrations: a systematic review and meta-analysis of 43 randomized controlled trial arms. Atherosclerosis. 2016;253:194-208.

28. Bedimo RJ, Mar H, Bosch RJ, Drechsler H, Cyktor JC, Macatangay BJC, et al. Brief report: no evidence for an association between statin use and lower biomarkers of HIV persistence or immune activation/inflammation during effective ART. J Acquir Immune Defic Syndr. 2019;82:e27-31.

29. Eckard AR, Jiang Y, Debanne SM, Funderburg NT, Mccomsey GA. Effect of 24 weeks of statin therapy on systemic and vascular inflammation in HIV-infected subjects receiving antiretroviral therapy. J Infect Dis. 2014;209:1156-64.

30. Mazidi M, Karimi E, Rezaie P, Ferns GA. Treatment with GLP1 receptor agonists reduce serum CRP concentrations in patients with type 2 diabetes mellitus: a systematic review and meta-analysis of randomized controlled trials. J Diabetes Complicat. 2017;31:1237-42. https://doi.org/10.1016/j. jdiacomp.2016.05.022.

31. Borges ÁH, O'Connor JL, Phillips AN, Rönsholt FF, Pett S, Vjecha MJ, et al. Factors associated with plasma IL-6 levels during HIV infection. J Infect Dis. 2015;212:585-95. https://doi.org/10.1093/infdis/jiv123.

32. Nordell AD, McKenna M, Borges ÁH, Duprez D, Neuhaus J, Neaton JD. Severity of cardiovascular disease outcomes among patients with HIV is related to markers of inflammation and coagulation. J Am Heart Assoc. 2014;3:e000844. https://doi.org/10.1161/JAHA.114.000844.

33. Kaplan RC, Landay AL, Hodis HN, Gange SJ, Norris PJ, Young M, et al. Potential cardiovascular disease risk markers among HIV-infected women initiating antiretroviral treatment. JAIDS J Acquir Immune Defic Syndr. 2012; 60:359-68. https://doi.org/10.1097/QAl.0b013e31825b03be.

34. Shive $\mathrm{CL}$, Jiang $\mathrm{W}$, Anthony DD, Lederman MM. Soluble CD14 is a nonspecific marker of monocyte activation. AIDS. 2015;29:1263-5. https://doi.org/10.1097/QAD.00000000000000735.

35. Lien E, Aukrust P, Sundan A, Müller F, Frøland SS, Espevik T. Elevated levels of serum-soluble CD14 in human immunodeficiency virus type 1 (HIV-1) infection: correlation to disease progression and clinical events. Blood. 1998; 92:2084-92 http://www.bloodjournal.org/content/92/6/2084.abstract.

36. Lederman MM, Funderburg NT, Sekaly RP, Klatt NR, Hunt PW. Residual immune Dysregulation syndrome in treated HIV infection. J Infect Dis. 2013; 191:51-83. https://doi.org/10.1016/B978-0-12-407707-2.00002-3.

37. Pussinen PJ, Havulinna AS, Lehto M, Sundvall J, Salomaa V. Endotoxemia is associated with an increased risk of incident diabetes. Diabetes Care. 2011; 34:392-7. https://doi.org/10.2337/dc10-1676.

38. Taylor BS, So-Armah K, Tate JP, Marconi VC, Koethe JR, Bedimo RJ, et al. HIV and obesity comorbidity increase interleukin 6 but not soluble CD14 or Ddimer. J Acquir Immune Defic Syndr. 2017;75:500-8.

39. Mave V, Erlandson KM, Gupte N, Balagopal A, Asmuth DM, Campbell TB, et al. Inflammation and change in body weight with antiretroviral therapy initiation in a multinational cohort of HIV-infected adults. J Infect Dis. 2016; 214:65-72.

\section{Publisher's Note}

Springer Nature remains neutral with regard to jurisdictional claims in published maps and institutional affiliations.

\section{Ready to submit your research? Choose BMC and benefit from}

- fast, convenient online submission

- thorough peer review by experienced researchers in your field

- rapid publication on acceptance

- support for research data, including large and complex data types

- gold Open Access which fosters wider collaboration and increased citations

- maximum visibility for your research: over $100 \mathrm{M}$ website views per year

At BMC, research is always in progress.

Learn more biomedcentral.com/submissions 